\title{
Reasoning Under Uncertainty: The Role of Two Informal Fallacies in an Emerging Scientific Inquiry
}

\section{Louise CUMmings}

Nottingham Trent University
Résumé: C'est maintenant une pratique courante de reconnaitre que plusieurs des sophismes traditionnels sont des arguments raisonnables. C'est l'examen des sophismes traditionnels dans leur plus large contexte d'usage qui a principalement contribué à ce changement d'évaluation. Toutefois, ce virage pragmatique dans l'évaluation des sophismes ne fait que débuter. Le potentiel de cette approche n'est pas encore pleinement exploré. J'examine comment certains sophismes traditionnels accordent des gains épistémiques à des recherces dans le contexte d'investigation scientifique. Je soutiens que ces sophismes facilitent le progrès des investigations, surtout dans leurs premières étapes lorsque l'incertitude domine le contexte épistémique. II découle de mes réflexions que ces sophismes sont des raisonnements de découvertes dans les contextes d'incertitude épistémique.

Keywords: Argument from analogy, argument from ignorance, bovine spongiform encephalopathy, context, Douglas Walton, epistemic uncertainty, fallaciousness/nonfallaciousness and scientific inquiry.

\section{Bovine spongiform encephalopathy: some background}

Bovine spongiform encephalopathy (BSE) is a progressive, degenerative disease of the brain in cattle. Since its widespread emergence in British cattle in the 1980s, its implications for animal and human health have been both devastating and, in key respects, essentially unknown. Many thousands of cattle have developed the disease (clinical cases). In early 1993, cases were being reported at a rate of around 1,000 a week.' Thousands of other animals who were incubating the disease in the absence of clinical signs-subclinical cases-are believed to have entered the human food chain. BSE subclinical cases have now been linked to the 
emergence of a new human spongiform encephalopathy - new variant CreutzfeldtJakob disease (nvCJD). ${ }^{2}$ Notwithstanding the identification of the source of nvCJD, there is much that remains unknown about this disease and about BSE itself. The inquiries into both of these diseases are still in their initial stages. The lack of knowledge that attends any inquiry in its early stages makes a case study of the inquiry into BSE a fertile ground indeed in which to test the validity of the claim that certain informal fallacies constitute rationally acceptable heuristics of reasoning in contexts of epistemic uncertainty.

\section{BSE, uncertainty and lack of knowledge}

Even a cursory examination of the BSE Inquiry report reveals something of the extent to which both scientists and government ministers were confronted with a lack of knowledge and of certainty on the issue of BSE. For example, in the covering minute of a submission that was forwarded to Mr. Edward Smith, the Deputy Secretary at the Ministry of Agriculture, Fisheries and Food (MAFF), Mr. Cruickshank of MAFF's Animal Health Group remarked:

We do not know where this disease came from, we do not know how it is spread and we do not know whether it can be passed to humans. The last point seems to me the most worrying aspect of the problem. (BSE Inquiry, Volume 1, p. 44)

Mr. Cruickshank's concern about the transmissibility of BSE to humans reflected the centrality of this issue in the Government's response to BSE, a response which took place against a 'background of uncertainty':"3

One of the most significant features of BSE and other TSEs [transmissible spongiform encephalopathies] is the fact that they are diseases with very long incubation periods. Thus the question whether BSE was transmissible to humans was unlikely to be answered with any certainty for many years, and scientific experiments were bound to take a long time. The Government had to deal with BSE against this background of uncertainty as to the transmissibility of the disease. (BSE Inquiry, Volume 1, p. xx)

The uncertainty and lack of knowledge that attends the issue of BSE has had a variable impact on the policies of successive British governments in the area. In some quarters, lack of knowledge was taken to justify a policy of 'no action':

In view of our very uncertain knowledge of the disorder it does not seem appropriate at this stage for MAFF to issue general information other than, perhaps, of a technical nature in a publication such as the Veterinary Record. (BSE Inquiry, Volume 3, p. 25)

Meanwhile, with our current incomplete state of knowledge no action by MAFF is recommended beyond attempting to ensure that publicity is wellinformed and not unduly alarmist. (BSE Inquiry, Volume 3, p. 34$)^{5}$

In other quarters, uncertainty and lack of knowledge warranted the taking of various measures that were designed to protect animal and human health: 
Given that there were so many uncertainties, a complete withdrawal of the MBM [meat and bone meal] material from feed for ruminants was considered to be the only safe solution. (BSE Inquiry, Volume 3, p. 81) ${ }^{6}$

In the absence of knowledge, the ruminant feed ban should have been implemented on a 'worst case' assumption. (BSE Inquiry, Volume 3, p. 117)'

The main reason for introducing a policy of slaughter with compensation would be to safeguard public health in the absence of knowledge about possible transmissibility to humans. (BSE Inquiry, Volume 3, p. 128)

The Working Party concluded, and rightly concluded, that it was not safe to proceed on the basis that it was certain that BSE would not transmit orally to humans. They concluded that action was called for to meet the risk that BSE would not behave like scrapie. They were driven to that conclusion by uncertainty. (BSE Inquiry, Volume 4, p. 57)

That BSE presented scientists and government ministers alike with a crisis of certainty is beyond doubt. From questions of origin to questions of transmission, knowledge of BSE was essentially lacking. Yet with a rapidly growing number of animal cases and with the potential for transmission to humans great indeed, there was an urgent need to bridge this lack of knowledge. Unsurprisingly, 'parallels' began to be forged with other encephalopathies; forms of 'guesswork' were instituted. ${ }^{10}$ Probabilities swiftly replaced scientific certainties as the epistemic standard of decision-making in questions of policy. ${ }^{11}$ It is against this straightened epistemic background, I want to contend, that certain traditional informal fallacies emerge as rationally acceptable or non-fallacious modes of argument. In the next section, I examine two such modes of argument - the argument from ignorance and the argument from analogy. I describe the main features of each of these arguments, using as my examples extracts of reasoning from the BSE Inquiry report. In Section 4, I examine the epistemic contribution of each argument to the process of BSE inquiry in particular and of scientific inquiry in general. The model of reasoning that emerges places informal fallacies at the centre of rational methodology in science. I conclude with a discussion of the implications of this model for the future direction of fallacy inquiry.

\section{BSE and two informal fallacies}

Motivated by an impending sense of crisis, scientists and government ministers were not long in developing strategies of reasoning that were essentially adaptive to the lack-of-knowledge context in which they found themselves operating. Two types of argument or reasoning were especially significant in this regard. The first type - the argument from ignorance - was employed with such force and consistency that it came to be labelled by the BSE Inquiry Team as the 'mantra' of the BSE story. Its manifestations were numerous. Most commonly, it consisted in 'no evidence' claims of the following type: 
On 15 October [1987] Mr, Suich circulated information in Question and Answer form to enable press officers and others to answer queries about BSE. This included:

Q. Can it be transmitted to humans?

A. There is no evidence that it is transmissible to humans.

(BSE Inquiry, Volume 3, p. 123)

Two variants of this form of the argument involve claims of 'no reason' and 'no (scientific) justification':

When Mr. Dorrell made his statement to Parliament, he was unable to answer an obvious question. Were children more susceptible than adults to BSE? All that he could say was that he had asked SEAC [Spongiform Encephalopathy Advisory Committee] to advise on this. In the event SEAC advised that there was no reason to believe that children were particularly susceptible. (BSE Inquiry, Volume 1, p. 164; emphasis added)

In our [SEAC's] judgement any risk as a result of eating beef or beef products is minute. Thus we believe that there is no scientific justification for not eating British beef and that it can be eaten by everyone. (BSE Inquiry, Volume 1, p. 131; emphasis added)

In relation to this form of the argument from ignorance, Schedler (1980) remarks: '... a reader assumes that "there is no reason to believe P" at least implies, or perhaps is another way of saying, "there is reason to believe P is false"...' (p. 70). Certainly in their media interactions with the general public, the implication that BSE was not transmissible to humans was an implication that government ministers and officials were content to have proceed uncorrected. ${ }^{12}$ Such an understanding of the transmissibility of BSE on the part of the public served to allay public anxiety about the disease and to protect a highly profitable meat industry. What is particularly remarkable about this form of the argument from ignorance is the extent to which it was employed by scientists who were called upon to investigate BSE and its implications for animal and human health (e.g., SEAC members) ${ }^{13}$ Experienced scientists within their respective fields of inquiry, these BSE inquirers were clearly aware that "it is part of the lore of experimental science that the want of confirming evidence for a hypothesis is not to be confused with disconfirming evidence' (Woods and Walton, 1978, p. 89). Their extensive use of this form of the argument from ignorance, I believe and will argue subsequently, is deeply revealing of the nature of rational scientific methodology itself.

A second form of the argument from ignorance featured prominently in inquiry into BSE. It is exemplified in the following remarks of the Inquiry Team:

No consideration appears to have been given to the risk that scrapie might be recycled in sheep, or even transmitted to other farm animals. This may seem surprising. The answer probably lies in the fact that half a century had elapsed without any indication that animal feed containing ovine protein was infecting sheep or any other animal. (BSE Inquiry, Volume 1, p. 227) 
The reasoning of this extract can be reconstructed as follows:

If animal feed containing ovine protein could infect sheep or other animals, then it would have done so in half a century.

Such infection had not occurred in half a century.

Therefore, animal feed containing ovine protein cannot infect sheep or other animals. ${ }^{14}$

This reconstruction serves to make explicit what Walton (1996) has described as the 'three components' of the argument from ignorance - a modus tollens inference, an ignorance premise and a search premise. ${ }^{15}$ While the validity of the modus tollens inference is beyond dispute, the ignorance premise (if animal feed containing ovine protein ...) and the search premise (such infection had not occurred ...) are essentially contestable. Their contestable nature stems from the fact that these premises can be more or less warranted in certain contexts of use. The variable warranty of these premises is often at the centre of an analysis of the rational merits of the argument from ignorance.

A second argument that came to characterise scientific inquiry into BSE was the argument from analogy. Typically, this argument consisted in a comparison of BSE with scrapie, a spongiform encephalopathy that had been endemic in the sheep population of Britain for some 250 years. ${ }^{16} \mathrm{I}$ will examine subsequently the issue of the rational acceptability of this particular argument type. In the meantime, I describe the various manifestations of this argument within scientific inquiry into BSE.

I described above how scientists inquiring into BSE were confronted, at least initially, with an extensive lack of knowledge of all the main issues surrounding this disease, for example, the potential of the disease to transmit to humans, etc. Much of this lack of knowledge, I argued, is typical of any inquiry that is in its early stages of development. However, BSE presented scientists with a unique problem of knowledge acquisition, a problem that threatened to prolong scientific uncertainty for a considerable and excessive period of time. Transmissible spongiform encephalopathies were known to have a long incubation period, in most cases of several years. ${ }^{17}$ In view of this fact, transmission studies and tissue infectivity tests ${ }^{18}$ would take many months and years to fully implement, even if undertaken with the greatest urgency. Against this evidentially bereft epistemic background it became necessary to draw upon prior knowledge of TSEs in general, and of scrapie in particular, ${ }^{19}$ in making various policy decisions:

$\ldots$ in the absence of a test for the BSE agent, and before results of tissue infectivity tests were available for BSE, any decision to exclude specific tissues from the human food chain could only be based on experience with other TSEs. This essentially meant that the justification for the SBO [Specified Bovine Offal] ban of November 1989 was based on work with scrapie. (BSE Inquiry, Volume 2, p. 115)

In this way, specific analogies with scrapie were developed, some of which related to the safety of cattle tissues for human consumption and of human-based 
products in medical treatments, others of which related to the question of the transmissibility of BSE to humans:

He [Dr Kimberlin] ... said that he was not overly concerned about the thymus because scrapie research indicated that thymus was lower risk than other LRS [lymphoreticular system] tissues. (BSE Inquiry, Volume 1, p. 117) ${ }^{20}$

The Chairman of the CSM [Committee on Safety of Medicines], Professor Sir William Asscher, told us that experience with human growth hormone and dura mater implants had made the Committee very wary of parenteral products. However, the fact that scrapie had not transmitted to man gave reassurance that BSE was unlikely to be acquired orally. (BSE Inquiry, Volume 1, p. $173)^{21}$

On 9 February 1989 they [the Southwood Working Party] submitted a Report to the Government in the knowledge that it would be published. The report concluded that the risk of transmission of BSE to humans appeared remote and that 'it was most unlikely that BSE would have any implications for human health.' This assessment of risk was made on the following basis: BSE was probably derived from scrapie and could be expected to behave like scrapie. Scrapie had not been transmitted to humans in over 200 years and so BSE was not likely to transmit either. (BSE Inquiry, Volume 1, p. $\mathrm{xx})^{22}$

A reconstruction of the reasoning of the last quotation above produces the following argument:

BSE is similar to scrapie in certain respects (analogy premise).$^{23}$

Scrapie is not a zoonosis. ${ }^{24}$

Therefore, BSE is not a zoonosis.

The structure of this argument is typical of that of an argument from analogy. Two entities (in this case, diseases), are shown to be similar; it is then argued that since one entity has a property $\mathrm{P}$, the other entity will also have that property (in this case, $\mathrm{P}$ is the property of not being a zoonosis). ${ }^{25}$ What makes this particular use of the argument fallacious is the lack of warranty that attends the analogy premise. (It was established in 1987 that scrapie was not a zoonotic condition, ${ }^{26}$ so the second premise of the argument above is essentially warranted). Even by 1989 , considerable research had been amassed that indicated that BSE and scrapie were distinct disease entities. It had already been demonstrated that BSE had a greater host range than scrapie. ${ }^{27}$ The transmission properties ${ }^{28}$ of BSE and its pathogenesis ${ }^{29}$ (temporal spread of infectivity in a host) were also known to differ significantly from those of scrapie. At the same time as these findings served to reveal important dissimilarities between BSE and scrapie, the epidemiological evidence that was generally accepted ${ }^{30}$ to support the analogy premise should properly have been accorded less significance than the results of biological studies. BSE was first brought to the attention of Mr. John Wilesmith, Head of the Epidemiology Unit at the Central Veterinary Laboratory, in late May 1987, when Dr. William Watson, Director of the CVL, asked him to investigate the epidemiology of the new disease. Mr. Wilesmith concluded that it was most unlikely that BSE was 
derived from existing strains of scrapie and that:

The source of infection ... was tissues derived from sheep infected with conventional scrapie [in $\mathrm{MBM}^{3}$ ]; the MBM had become infectious because rendering methods which had previously inactivated the conventional scrapie agent had been changed. (BSE Inquiry, Volume I, p. xix)

Against our current knowledge of BSE and scrapie, ${ }^{32}$ the Inquiry Team described these conclusions as 'reasonable but fallacious. ${ }^{33}$ Yet, given that the results of various biological studies were already known in 1989, results which told strongly against a scrapie origin of BSE, these conclusions lacked rational warrant at a much earlier stage of the BSE story than that recognised by the Inquiry Team. Throughout the BSE epidemic, epidemiological studies played a vital role in monitoring the incidence and distribution of BSE and in establishing the effectiveness of various measures that were designed to bring the disease under control. Notwithstanding this fact, epidemiological findings function in inquiry as a type of default evidence, that is, evidence that stands in the absence of other evidence that exhibits greater rational warranty.

That a gradation of types of evidence existed in scientific inquiry into BSE is suggested by the following statements:

[I]t was inevitable that concerns about infection via cross-contamination of feed grew progressively as more BABs [cattle born after the ban] were confirmed and other sources of infection were not identified. The introduction of the ELISA test in June 1994 provided confirmatory evidence soon after that cross-contamination was a real issue that had to be dealt with. In other words hard evidence was found as opposed to anecdotal evidence and interpretation of epidemiological data. (BSE Inquiry, Volume 5, p. 83; emphasis added) ${ }^{34}$

MAFF advised that there was epidemiological proof rather than hard evidence that cross-contamination took place at a particular mill on a particular day. (BSE Inquiry, Volume 5, p. 118; emphasis added) ${ }^{35}$

Epidemiological evidence, it emerges clearly, should have assumed a secondary evidential role to the findings of the various biological studies that were conducted into BSE, studies that revealed differences in the host range, transmission properties and pathogenesis of scrapie and BSE. The fallaciousness of the scrapie analogies examined previously, then, consists in the failure of scientists and government officials to accord different levels of rational warranty to different types of evidence, here exemplified by the use of epidemiological evidence to support the inferences of these analogical arguments when available biological evidence effectively weakened these inferences.

\section{The argument from analogy and the argument from ignorance: epistemic contribution to inquiry}

The rational merits of the argument from ignorance are by now well documented. It has long been recognised, for example, that within a court of law, the legal 
presumption of innocence is none other than a non-fallacious argument from ignorance: ${ }^{36}$

This mode of argument is not fallacious in a court of law, because there the guiding principle is that a person is presumed innocent until proven guilty. (Copi, 1961, p. 57)

The defense can legitimately claim that if the prosecution has not proved guilt, this warrants a verdict of not guilty. (Copi, 1972, p. 77)

In still other contexts, this argument is not only non-fallacious, but is deductively valid..$^{37}$

To the extent we know a knowledge-based $\mathrm{K}$ is closed, i.e., complete, in the sense of containing all the relevant information, we can infer that if a proposition $\mathrm{A}$ is not in it, then $\mathrm{A}$ is false. This argumentation scheme for the argumentum ad ignorantiam has the following form:

All the true propositions in domain $\mathrm{D}$ of knowledge are contained in $\mathrm{K}$.

$A$ is in $D$.

$A$ is not in $K$.

For all $A$ in $D, A$ is either true or false.

Therefore, $\mathrm{A}$ is false.

This form of inference is deductively valid (Walton, 1992, pp. 385-386).

However, notwithstanding the attempt to describe the rational features of the argument from ignorance within different contexts of use, little has been written to date about the probative role of this argument within the setting of an inquiry. ${ }^{38}$ Indeed, as inquiry is standardly characterised in the literature, a probative role for this argument is effectively precluded. For most theorists an inquiry embodies a hierarchical process of reasoning, in which inquirers reason from premises that are well known or well established to a conclusion that is less well known or less well established:

The inquiry is a collaborative investigation that seeks to prove something, or alternatively to show that the existing evidence is insufficient to prove it. The inquiry is a hierarchical procedure of reasoning (similar to an Aristotelian demonstration) where the premises are supposed to be better known or established than the conclusion to be proved from them. (Walton 1990, p. 414)

The epistemic progression of an inquiry - reasoning from what is known to what is unknown-precludes the use of non-hierarchical argumentative strategies within inquiry. These non-hierarchical strategies are often typical of the reasoning of informal fallacies, Question-begging argument, with its essentially circular pattern of demonstration, is a case in point. Here one reasons from premises that are as unknown as the conclusion-to-be-proved, by virtue of the fact that the premises and conclusion are the same proposition..$^{39}$ The epistemic hierarchy of inquiry is again not evident in the argument from ignorance, where both the premises and the conclusion are of the order of presumptions. As Walton (1992) remarks: 
Not only is it right to say that the argumentum ad ignorantiam is closely linked to presumptive reasoning and burden of proof. You could even say that the very structure of the argumentum ad ignorantiam is an expression of how presumptive reasoning and burden of proof can function correctly in argumentation to shift a presumption to the other side in a dialogue. (p. 386; emphases in original. $)^{40}$

Notwithstanding significant differences in the epistemic structures of question-begging argument and the argument from ignorance on the one hand and of inquiry on the other hand, both of these arguments have been documented to function non-fallaciously in a range of inquiries. ${ }^{41}$ What this suggests, I believe, is not that we need to develop further, more sophisticated ways of proscribing certain argument sequences in inquiry (à la Rescher, 1977), but that we need to extend our notion of inquiry to include argument structures that are, amongst other things, circular and presumptive ${ }^{42}$ in nature. By expanding the notion of inquiry, two main gains are achieved. Firstly, the conception of inquiry that we employ in our theorising on matters of argumentation more closely reflects the epistemic contexts in which scientists operate. It is seldom, if ever, the case that scientists have access to complete knowledge within their investigations. Rather, scientists are more often compelled to reason within the lack-of-knowledge contexts that were discussed above in relation to BSE. An inquiry that assumes a hierarchical structure, in which reasoning proceeds linearly from propositions that are well known to propositions that are less well known, is ill-equipped to accommodate the reasoning strategies that occur in contexts of knowledge deprivation and epistemic uncertainty. Secondly, within an expanded conception of inquiry arguments like question-begging argument and the argument from ignorance can be characterised not only as non-fallacious, but also as constitutive of the rational methodology of inquiry in contexts of uncertainty and lack of knowledge. Both of these gains are central motivations for my subsequent analysis of ignorance and analogical arguments in inquiry into BSE.

Consider the following extracts from the BSE Inquiry:

In response to intense media coverage, Dr Calman released a statement on 26 January [1994]. This stated that:

- no one knew what illness the patient was suffering from; and

- on the basis of the work done so far, there was no evidence whatever that BSE caused CJD and, similarly, not the slightest evidence that eating beef or hamburgers caused CJD. (BSE Inquiry, Volume 1, p. 143) (33 $^{4}$

On 14 February 1992 BSE was found to have been successfully transmitted to a marmoset by cerebral inoculation. This was the first transmission to a primate. A meeting of SEAC was immediately called to consider the implications of this. SEAC concluded that as marmosets had in the past been infected with SEs, including scrapie, using similar methods, the results were not surprising and had no implications for the safeguards already in place for human and animal health. (BSE Inquiry, Volume 1, p. 139) 
The first extract above is typical of the argument from ignorance reasoning that, I claimed earlier, dominated the BSE story. The clear implication of Dr. Calman's statement that there is no evidence that BSE causes CJD is that BSE does not cause CID. The second extract is representative of the analogical reasoning that frequently characterised inquiry into BSE. Like scrapie before it, BSE had been transmitted to a marmoset. Given that BSE shared certain transmission properties with scrapie, it could be expected to share with scrapie one further transmission property, that of failing to transmit to humans. Both of these conclusions-that BSE does not cause CJD and that BSE does not transmit to humans-are now known to be incorrect. ${ }^{44}$ However, the fact of their incorrectness can play no part in the rational evaluation of the arguments that gave rise to these conclusions. Within this evaluation, an assessment of the fallaciousness or otherwise of these arguments can only be based upon what was known about BSE at the time that these arguments were made. ${ }^{45}$ It is against this knowledge background, I contend, that both the argument from ignorance and the argument from analogy performed a significant epistemic function, that of facilitating inquiry in a context of lack of knowledge and uncertainty.

Given the very obviously appropriate ethical constraints that surround the use of human subjects in scientific experiments, the question of whether or not BSE could be transmitted to humans was only ever going to be definitively answered by the emergence of a previously unidentified spongiform encephalopathy in the human population. Indeed, there is a very real sense in which the answer to this question of transmission would still be unknown had the current total of 105 deaths from nvCJD never occurred. The long incubation period of spongiform encephalopathies would probably have precluded the answering of this question for many years to come. Scientists, who were mindful of the impossibility of directly answering this question of transmission, had little option but to accord it a low epistemic priority or no epistemic priority at all within their deliberations, and to institute in its place questions that were, by their very nature, susceptible of investigation. In this way, early research into BSE concentrated on establishing the incidence and distribution of the disease and on ascertaining if BSE exhibited similarities to previously identified animal and human spongiform encephalopathies. These microbiological ${ }^{46}$ and epidemiological ${ }^{47}$ studies sought to address specific research questions, questions for which an answer was at least possible in the then available epistemic context. However, the cumulative knowledge that was obtained through these studies and many other studies besides could at best provide an indirect answer ${ }^{48}$ to the question of whether or not BSE was transmissible to humans. With no answer to this question of transmission possible, short of the emergence of a new spongiform encephalopathy in humans, to inquire into this question was to disregard other vital research priorities. Arguing from a position of ignorance of the proposition that BSE transmits to humans to the falsity of that proposition had the significant epistemic function of removing the question of transmission from further inquiry. This last claim warrants examination. 
Not all the questions that emerged in inquiry into BSE were of the same epistemic standing. For questions such as 'Is BSE a form of scrapie in cattle?', an answer was in practice at least possible and was sought directly through a series of epidemiological, microbiological and histopathological investigations..$^{49}$ For the question 'Is BSE transmissible to humans?', no definitive answer would be forthcoming in the absence of the actual emergence of a new spongiform encephalopathy in humans and any investigations into this question were necessarily indirectly based, for example, on inferences from transmission work on primates. The potential of each of these questions to be answered within an inquiry effectively determines their epistemic order within that inquiry. Questions that can be addressed only indirectly, by inferences from the answers to other questions, cannot be epistemically prior to these other questions. Within the process by means of which questions are ordered in inquiry, the argument from ignorance functions, I contend, by excluding from inquiry any thesis or claim for which direct evidence is not attainable or is unlikely to be attainable in the short or longer term. Exclusion can only be achieved by representing a thesis as confirmed or disconfirmed and, thus, as not requiring further investigation within inquiry; a thesis which is unconfirmed is subject to continuing deliberation in inquiry. The pattern of confirmation in which theses are either confirmed or disconfirmed but, importantly, not unconfirmed, is essentially the pattern of confirmation that underlies the argument from ignorance.$^{50} \mathrm{By}$ arguing from a lack of evidence for the claim that BSE transmits to humans to the disconfirmation of that claim, an inquirer removes the (essentially unanswerable) question of BSE transmission to humans from further inquiry. In doing so, s/he makes a significant epistemic gain, that of according epistemic priority to the answerable questions about BSE.

The argument from ignorance, I am claiming then, is essentially non-fallacious in the context of an emerging scientific inquiry. In any new inquiry the need to establish knowledge is urgent. Knowledge can be acquired most efficiently by prioritising the questions that are investigated within inquiry, such that those questions for which answers are most immediately attainable assume epistemic priority over other, less immediately answerable questions. The argument from ignorance functions within such an epistemic context by foreclosing inquiry into those questions for which an answer is unlikely to be forthcoming in the short or longer term or for which an answer is dependent on the answers to yet other questions. However, in conferring epistemic priority on some questions and foreclosing inquiry into certain other questions, the argument from ignorance is not serving to exclude unimportant questions from inquiry. (Indeed, a question into which inquiry was foreclosed - the question of whether or not BSE could transmit to humans-was the most important question of the BSE story.) Rather, the function of this argument in an inquiry is the strictly epistemic one of grading questions according to their potential to be answered in that inquiry. And this epistemic function, I have been arguing, is undertaken as part of the rational methodology of scientific inquiry. 


\section{Louise Cummings}

The argument from ignorance, I have been arguing, performs the significant epistemic function of ordering questions that are to be examined within scientific inquiry. I now want to argue that the argument from analogy functions within inquiry by recommending from the outset certain questions for investigation. I described above how an analogy with scrapie in sheep came to inform both scientific and policy thinking on matters relating to BSE. I argued that this analogy was fallacious in respect of its privileging of epidemiological evidence over the evidence of various biological studies, studies which revealed essential dissimilarities between BSE and scrapie. However, I want to contend that this same analogy with scrapie suggested to investigators key questions about BSE and possible lines of inquiry in relation to the disease. To appreciate the extent to which an analogy with scrapie influenced the development of research questions into BSE, one need only consider the range of issues upon which this analogy was brought to bear. ${ }^{51}$ In December 1988, Mr. Bradley of the Central Veterinary Laboratory produced an updated paper on the CVL's research and development programme, in which he outlined the aims of the CVL programme, the work that was already taking place and proposed work. Five experiments were already in progress, addressing questions in the areas of epidemiology, clinico-pathological studies, transmission, molecular biology and molecular genetics. In all five areas research was predicated upon similar investigations that had been conducted into scrapie. Epidemiological studies sought to establish the natural transmission routes of BSE, both from dam to offspring (vertical or maternal transmission) and from animal to animal (horizontal or lateral transmission). Both routes of transmission were known to occur in scrapie..$^{52}$ In establishing the histopathology of BSE, clinico-pathological studies engaged in a form of differential diagnosis with scrapie. ${ }^{53}$ Transmission studies examined the transmissibility of BSE to hamsters, calves, marmosets, mice, goats, mink and sheep, all of which were known to be susceptible to scrapie. Further transmission experiments investigated BSE infectivity of placenta (known to be infective for scrapie), semen and embryos, as well as of other tissues. Studies in molecular biology and in molecular genetics investigated, respectively, the presence of scrapie-associated fibrils (SAFs) in BSE and the role of genetic factors in the expression of BSE in cattle. As was the case with epidemiological, clinicopathological and transmission studies, experimentation in both of these areas was guided by analogical reasoning with scrapie--the prior identification of SAFs in scrapie-affected sheep and the role of the sip gene in controlling the incubation period of scrapie in sheep. In short, so numerous and specific were the questions and lines of inquiry that were generated by analogical reasoning with scrapie that this analogy effectively set into motion an entire programme of research for the BSE scientists. It remains for me to examine how such a programme of research contributed positively to the epistemic progression of inquiry.

The ability to ask pertinent and significant questions in an inquiry already presupposes an extensive knowledge base. For example, the question 'Does BSE have the same histopathology as scrapie?' presupposed a knowledge of the his- 
topathology of scrapie on the part of the scientists who asked this question. It follows that in order to develop a research programme-which is, in effect, a collection of such questions-inquirers must already have an extensive body of knowledge at their disposal. Yet, in the initial stages of any inquiry, such a body of knowledge is noticeably lacking. Under conditions of knowledge deprivation, analogical reasoning, I contend, functions by generating a framework of questions that has at least a degree of plausibility. As inquiry proceeds, some of these questions will be shown to be warranted, largely through a demonstration of the truth of the answers that they elicit. Other questions, that are less successful in eliciting true answers, will emerge from the process of inquiry as unwarranted. For BSE scientists, reasoning by analogy with scrapie represented a best attempt at generating research questions into BSE under conditions of uncertainty and lack of knowledge. At the initial stage of inquiry into BSE, this reasoning strategy had little in the way of rational validation to recommend it. Rather, the rational validation and invalidation of this reasoning strategy and of the questions that it generated came about as inquiry itself proceeded. ${ }^{54}$ The type of validation that is at issue in this context is demonstrated by the following statement from the BSE Inquiry Report:

The advances in knowledge by September 1994 significantly altered the scientific evaluation of the risk that BSE might be transmissible in humans.

Professor John Collinge told us:

Certainly the appearance in domestic and captive wild cats was a very important development. It demonstrated that you could no longer really plausibly argue that BSE was just scrapie in cows with all the same properties. This agent, wherever it had originated from, had quite different biological properties to scrapie as manifested by the extended host range of affected species, including things like nyala and kudu as well as the cats that had not been affected by scrapie before, so far as we were aware. (BSE Inquiry, Volume 1, p. 140)

The emergence of new knowledge in inquiry about the host range of BSE necessitated a re-evaluation of the rational standing of arguments that were based on an analogy with scrapie. These analogical arguments, for which scientists could 'no longer really plausibly argue', lost their epistemic status as plausible strategies of reasoning. In effect, their rational invalidation had been secured through the process of inquiry itself. At an earlier stage in inquiry into BSE, other scientific findings were taken to rationally validate the very same strategy of analogical reasoning. ${ }^{56}$ As findings from inquiry rationally validate and invalidate the strategy of analogical reasoning, the questions that are generated by this analogy are likewise validated and invalidated. Validated questions gain in epistemic stature and provide the basis of further lines of inquiry. Invalidated questions lose even their claim to plausibility within inquiry.

The argument from ignorance, I argued above, performed the significant epistemic function of prioritising research questions in inquiry into BSE. I am now claiming that the argument from analogy served an equally significant epistemic 
function in BSE inquiry, that of generating the questions that were to be addressed by inquiry. Both of these arguments are essentially fallacious according to traditional conceptions of the fallacies. The argument from ignorance is fallacious, it is argued, through its basing conclusions on claims of 'no evidence'; the argument from analogy is fallacious when there is evidence to indicate that a particular similarity does not hold. Yet both of these arguments, I have contended, constitute rationally acceptable strategies of reasoning in contexts of uncertainty and lack of knowledge in scientific inquiry. Indeed, it is just this context of scientific inquiry that, I believe, holds the best promise of understanding the non-fallaciousness of many of the so-called informal fallacies. In the next and final section, I examine briefly why I consider this to be the case. Central to that examination will be my earlier claims that not all of scientific inquiry proceeds in the strictly hierarchical fashion that is routinely depicted by theorists of argumentation and that many of the informal fallacies embody a non-hierarchical pattern of reasoning.

\section{Future research into the informal fallacies}

Of all the contexts in which reasoning and argumentation can be studied, scientific inquiry has received relatively little in the way of direct examination. Part of the reason for this neglect lies almost certainly with the technical nature of the questions that are examined within scientific inquiry. An evaluation of the reasoning of an inquiry presupposes knowledge of the subject matter that is addressed by that inquiry. However, another part of the reason lies in the fact that it is generally accepted that there is nothing new to say about the structure of scientific inquiry. Scientific inquiry has been almost universally characterised as a form of demonstration of a type which is Aristotelian or Euclidean ${ }^{57}$ in nature and this view of science is by now so well received that it does not occur to theorists to challenge it. One theorist to whom such a challenge has occurred is Nicholas Rescher. Many of Rescher's theoretical deliberations converge directly on the views that I have expressed above. For example, Rescher is keenly aware of the impact of uncertainty on the structure of the reasoning process $(1987,28)$ :

Much, if not most, of our thinking is carried out under conditions where we do not deem the premises from which we reason to be absolutely certain truths, but merely very probable or plausible suppositions. This situation has far-reaching implications for the appropriate character and structure of our reasoning, implications which are generally unheeded and unrecognized.

Moreover, the pattern of validation that I described earlier in relation to the questions that are generated through analogical reasoning - plausible questions being retrospectively validated by inquiry and assuming higher epistemic levels as a result of this validation - has a precedent in the method of retrospective reappraisal in Rescher's coherentist epistemology ${ }^{58}$ Importantly in the present context, Rescher's coherentism is a framework for scientific and inductive reasoning. Clearly, Rescher undertakes to interrelate issues of certainty and plausibility with struc- 
tures of reasoning and modes of inquiry, scientific inquiry specifically included. In doing so, his approach is not only unique in the literature on reasoning and argumentation, but is also directly pertinent to my own attempt to characterise traditional informal fallacies as acceptable strategies of reasoning in the context of scientific inquiry. For at the centre of that attempt are claims that are largely Rescherian in nature. I contend, for example, that uncertainty, rather than certainty, often characterises the process of inquiry in science, and that this uncertainty impacts on the structure of scientific reasoning through the resulting employment of patterns of argumentation that are typical of the informal fallacies. More ultimately, it is my contention that the informal fallacies are constitutive of at least part of the rational methodology of science. Herein lies, I believe, a guiding principle for future research into the fallacies.

Viewing the informal fallacies as part of the rational procedure of science promises gains for fallacy inquiry beyond those associated with more standard forms of analysis. ${ }^{59}$ Standardly, fallacy theorists proceed in analysis by examining a single type of argument across a range of different contexts. In some of these contexts the chosen argument will be shown to function fallaciously; in other contexts, the same argument will be seen to function non-fallaciously. A conclusion is then drawn about the rational features of the particular argument under examination, a conclusion which amounts to little more than a listing of fallacious and non-fallacious contexts of use. Moreover, such is the specificity of the analysis to one particular argument that the conclusion which is based on this analysis cannot be generalised to other arguments. So standardly in fallacy analysis what we end up with is a series of argument-specific contexts in which arguments may be used fallaciously and non-fallaciously. Conceptual similarities between argument types that effectively predispose them to be used within certain contexts and not to be used within other contexts-for example, their embodiment of presumptive reasoning-are lost from standard approaches with their emphasis on single-argument analysis. By reversing the direction of standard fallacy inquiry and beginning the analysis of arguments with an examination of context, we are less likely, I believe, to overlook significant conceptual similarities between the informal fallacies.

Just such an approach motivated my earlier analysis of the argument from ignorance and the argument from analogy. At the very centre of that analysis was an assessment of the epistemic attributes of a scientific inquiry in the early stages of its development. That assessment revealed the uncertainty of much of scientific inquiry, how inquiry was often compelled to proceed in the absence of knowledge and how both uncertainty and lack of knowledge came to impact on the structure of scientific reasoning. Having conducted this assessment of context, it was then possible to identify arguments that either directly embodied the epistemic features of scientific inquiry or provided a strategic reasoning response to those features. For example, I described above how the argument from analogy and the argument from ignorance bridged the lack of knowledge that characterised early scientific 
inquiry into BSE by, respectively, generating and prioritising research questions into the disease. With features of context effectively motivating the analysis of informal fallacies on this alternative approach, a unification of the fallacies based on their conceptual similarities is achieved, a unification which is not possible when the starting point of analysis is the single argument.

This alternative approach, moreover, is not without a precedent. Van Eemeren and Grootendorst place the notion of context, in the form of a critical discussion, at the centre of their pragma-dialectical analysis of the fallacies. ${ }^{60} \mathrm{However}$, where my own approach is unique is in its refusal to constrain in advance the context within which fallacies operate. Van Eemeren and Grootendorst pursue a speechact analysis of critical discussion. ${ }^{61}$ In doing so, they restrict from the outset their analysis of the fallacies to the conceptual choices that are possible within a speechact analysis of language. By refusing to employ a predetermined notion of context and by examining instead the actual epistemic contexts in which scientists operate, I have produced a form of fallacy analysis that is both reflective of scientific reasoning and deeply critical of received views of scientific inquiry.

\section{Notes}

'The source of this figure is the report of the BSE Inquiry, headed by Lord Phillips of Worth Matravers. For nearly three years Lord Phillips and his team examined all that was known about the history of BSE and nVCJD and looked at how these diseases were handled by the British Government and by others in the period between December 1986 and 20 March 1996. This report provides much of the factual background of this paper.

${ }^{2}$ On 20 March 1996, the then Secretary of State for Health, Mr. Stephen Dorrell, informed the British Parliament of the emergence of nVCJD and of the probable link of this disease to BSE in cattle.

${ }^{3}$ Not that there was any greater certainty or much more known about the transmissibility of BSE to cattle: '.. even the risk to cattle was not fully established; it was unknown whether BSE could infect cattle other than by some form of ingestion' (BSE Inquiry, Volume 1, p. 267).

${ }^{4}$ Submission from Mr. Rees (Chief Veterinary Officer, 1980-1988) to the Parliamentary Secretary, Mr. Donald Thompson, dated 5 June 1987.

s Submission from Mr. John Suich (MAFF Animal Health Division) to Mr. Donald Thompson, dated 7 July 1987.

"Summary comment on a meeting held on 18 May 1988 between Mr. Meldrum (Chief Veterinary Officer, 1988-1997) and Mr. John MacGregor (Minister of Agriculture, Fisheries and Food, 1987-1989), Mr. Donald Thompson and MAFF officials.

${ }^{7}$ Conclusion of the BSE Inquiry Team.

${ }^{8}$ Submission prepared by Mr. Suich in consultation with veterinary colleagues. Mr. Cruickshank minuted Mr. Edward Smith on 16 February 1988 with the submission to the Minister as prepared by Mr. Suich.

'Conclusion of the BSE Inquiry Team on the determinations of the Southwood Working Party, established 'to advise on the implications of Bovine Spongiform Encephalopathy and matters relating thereto' (BSE Inquiry, Volume 4, p. 2). 
${ }^{10}$ Sir Richard Southwood (Chair, Southwood Working Party) wrote on 9 August 1988 to Dr E. Poole of the Radcliffe Infirmary in Oxford, stating: 'my colleagues and I have made various recommendations based, I have to admit, largely on guesswork and drawing parallels from the existing knowledge of scrapie and CJ disease' (BSE Inquiry, Volume 4, p. 47).

"In a written statement to the BSE Inquiry, Sir Richard Southwood stated: "We were also conscious that there were uncertainties in virtually every aspect and that all we had to go on were analogies with scrapie in sheep and goats and kuru and CID in humans. We accepted that the agent seemed to be what was termed a 'slow virus' and therefore it could be a long time before the many necessary experiments would give results. Therefore these should be started as soon as possible, for until there was more knowledge, policy would have to be based on probabilities rather than scientific certainty' (BSE Inquiry, Volume 4, p. 1).

${ }_{12}$ A notable exception was the following statement of Dr. Kenneth Calman (Chief Medical Officer, 1991-1998) in a press release in October 1995 to mark the release of the fourth annual report of the CJD surveillance unit. Dr. Calman stated: ' 1 continue to be satisfied that there is currently no scientific evidence of a link between meat eating and development of CID and that beef and other meats are safe to eat. However, in view of the long incubation period of CJD, it is important that the Unit continues its surveillance of CJD for some years to come' (BSE Inquiry Report, Volume I: p. 149). The first sentence of this statement establishes the basis of an argument from ignorance-there is no scientific evidence of a link between meat eating and the development of CJD, therefore there is not a link between meat eating and the development of CJD. That this is the intended implication of these remarks is indicated by Dr. Calman's further claim that 'beef and other meats are safe to eat'. The force of the argument from ignorance is substantially weakened, however, by Dr. Calman's further remark about the long incubation period of CJD. This additional claim serves to highlight the fact that the absence of evidence of a link between meat eating and CID should not be taken to indicate that such a link does not exist.

${ }^{13}$ SEAC's judgement that 'there is no scientific evidence for not eating British beef and that it can be eaten by everyone' is an ignorance argument of the form ' $\mathrm{P}$ because there is no reason why not-P' (Robinson, 1971: p. 99).

"Collins, Aiello, Warnock and Miller (1975) describe a similar form of the argument from ignorance. A computer program called SCHOLAR is asked whether rubber is a product of Guyana: 'SCHOLAR does not have any specific item of knowledge saying that Guyana produces rubber or not. However, SCHOLAR does know that Peru and Colombia are the major rubber producers in South America. And SCHOLAR also knows that rubber is an important product, so if Guyana did produce rubber, SCHOLAR would presumably know it. SCHOLAR concludes: "I know enough that I am inclined to believe that rubber is not an agricultural product of Guyana" (p. 398). As with the example of the main text, the ignorance reasoning of this example can be reconstructed as follows: if Guyana did produce rubber, I would know it; I do not know that Guyana produces rubber; therefore, it is false that Guyana produces rubber (Guyana does not produce rubber). My discussion of the example in the main text is equally applicable to this case,

is Walton (1996) remarks of the argument from ignorance: 'The presence of some knowledge (usually incomplete), is combined with the absence of other knowledge (i.e., ignorance) to draw a conclusion about the significance of this lack of knowledge or missing knowledge. The inference takes a modus tollens form... if A were true (false), A would be known to be true (false) but $A$ is not known to be true, therefore $A$ is false (true). These three components, the ignorance premise, the search premise, and the modus tollens inference, characterise the argumentum ad ignorantiam as a distinctive species of argument' (p. 246).

${ }^{16}$ Although the first record of scrapie was made in 1732 , the first published article appeared in 1913 in the Journal of Comparative Pathology. 
17 Iatrogenic transmission of CID (transmission through some form of medical treatment or surgery) has permitted the calculation of specific incubation periods for this disease. 'Central inoculation through neurosurgery, depth electrodes, corneal graft or dura mater graft results in disease after a mean incubation period of about 2 yr .... Peripheral inoculation through human pituitary derived growth hormone (hGH) or pituitary derived gonadotrophin ( $\mathrm{hGnH}$ ) results in disease after an incubation period range from a minimum of $4 \mathrm{yr}$ to a maximum of at least $30 \mathrm{yr}$ with a mean of around 12 yr' (Will, 1993: p. 963).

${ }^{18}$ Tissue infectivity tests were vital not only in establishing the progression of disease in infected animals (which organs were infectious at different stages post-inoculation) but also in arriving at decisions about which cattle tissues to include in the Specified Bovine Offal ban of November 1989.

${ }^{19}$ The analogy with scrapie was particularly evident in the reasoning of BSE scientists. This is related to the fact that when BSE first emerged as a new disease entity, more was known about scrapie than about any other TSE. Scrapie research was already underway in the 1950 s when Zigas and Gajdusek first reported kuru, a human TSE, in the Fore people of Papua New Guinea.

${ }^{20}$ Dr. Richard Kimberlin was TSE research scientist at the Neuropathogenesis Unit in Edinburgh between 1981-88. Since 1988 he has been an independent TSE consultant. He was also a member of the Tyrell Committee, established in order to advise on research in relation to BSE, and a member of SEAC.

${ }^{21}$ A parenteral product is a product that is introduced into the body by some means other than by the mouth or bowel-for example, dura mater implants during surgery. It was already known that dura mater material could transmit CJD from person to person. So, the reasoning of this passage goes, if BSE was transmissible to humans, parenteral products would pose a BSE risk in the same way that they already posed a CJD risk.

${ }^{22}$ Scrapie analogies motivated decisions beyond those that are represented in the main text. For example, the decision to exclude cattle under six months of age from the ban was based on analogical reasoning with scrapie: 'Tissues from cattle aged under six months were exempt from the ban on the basis that scrapie infectivity had not been found in lambs of this age' (BSE Inquiry, Volume 1, p. 14); 'Analogy with scrapie research suggested that infectivity would not reach the brain or spinal cord of cattle in the first six months of life. This was a cogent argument for exempting brain and spinal cord of calves from the ban' (Volume 1, p. 116).

${ }^{23}$ This claim was often articulated more strongly as a claim of identification between BSE and scrapie-BSE was none other than 'bovine scrapie'.

${ }^{24}$ A zoonosis is any infection or disease that is transmitted to man from lower vertebrates.

${ }^{25}$ Of course, this argument is not restricted to two entities or situations but can encompass many situations: "An argument from analogy is not necessarily limited to two situations. If several situations can be shown to all share a particular characteristic, then it can be concluded that a new situation also shares that characteristic' (Walton, 1989: p. 258).

26. 'Extensive retrospective studies together with a review of world literature led to the conclusion, published in 1987, that scrapie had never passed to humans despite opportunities to do so over the 250 years during which the disease had contaminated sheepmeat entering the human food chain (BSE Inquiry, Volume 2, p. 67). The paper to which the Report makes reference was written by Brown, Cathala, Raubertas, Gajdusek and Castaigne.

27....[1]n February 1987, scientists at the CVL [Central Veterinary Laboratory] inoculated hamsters with BSE-infected bovine brain in an attempt to test for transmissibility. Further transmissions to hamsters were attempted at the CVL in January 1988. In the event, hamsters proved not to be susceptible to BSE, although they were readily susceptible to sheep scrapie' (BSE Inquiry, Volume 2, p. 68).

28 'The first demonstration of the transmissibility of BSE was in September 1988, when mice inoculated with BSE-infected brain material developed disease. This was important as it pro- 
vided an animal model with which to assay BSE infectivity. However, it was found that the incubation period for BSE in mice was shorter than for known scrapic isolates. While this finding was considered to demonstrate the potential of the mouse model for assaying infectivity, it also demonstrated a difference between the transmission properties of BSE and scrapie' (BSE Inquiry, Volume 2, p. 82).

29 'In 1982, Hadlow had studied the infectivity of various tissues from sheep affected with scrapie... He determined that after the brain and spinal cord, tissues of the lymphoreticular system (LRS)-including spleen, lymph nodes, intestinal Peyer's patches and tonsils-were the most infective... However, a similar study in cattle using the mouse bioassay... has shown that the spleen, lymph nodes and tonsils from BSE-affected cattle do not transmit disease. Of LRS tissues, only the distal ileum containing Peyer's patches has proved to be infective in 6month-old calves ... the patterns and extent of tissue infectivity in the two species are quite different' (BSE Inquiry, Volume 2, p. 84; emphasis added).

${ }^{30}$ This evidence was accepted by scientists, government officials and the public, although its increasingly dubious nature was never made clear to the public: 'The conclusion that BSE had been transmitted from scrapie-infected sheep was generally accepted... Although, as the years passed, evidence mounted that discredited the scrapie theory, this was never made clear to the public and most people are still under the impression that cattle caught BSE from scrapieinfected feed' (BSE Inquiry, Volume 1, p. 37).

${ }^{3 !}$ Meat and bone meal, a ruminant-derived protein supplement that is fed to cattle.

32 'These conclusions can be re-evaluated with hindsight, using what we now know about the cause of the disease and its biology' (BSE Inquiry, Volume 2, p. 74),

${ }^{33}$ BSE Inquiry, Volume 1, p. xix.

${ }^{34}$ Statement to the Inquiry Team by Dr. Matthews, Ministry of Agriculture, Fisheries and Food Senior Veterinary Officer, 1988-96.

${ }^{35}$ Note by UKASTA (UK Agricultural Supply Trade Association) of a meeting between UKASTA and MAFF representatives on 7 August 1995.

${ }^{36}$ The role of context in the positive evaluation of this argument is clearly emphasised in the following remarks of Walton: 'Whether an ad ignorantiam argument is reasonable often depends on the burden of proof as indicated by the context of dialogue. For example, the criminal law presumes that a person is not guilty if he has not been shown to be guilty. This is an ad ignorantiam form of argument, but it can be reasonable in the context of the rules of argument in criminal law' (Walton, 1989, p. 47).

${ }^{37}$ Walton describes these cases as 'rare' in 'actual practice'. It is more usually the case that "when an argument from ignorance is reasonable, it is a weak form of argument that depends on the context of dialogue' (Walton, 1989, p. 47).

${ }^{38}$ This is due in no small part to the fact that Frans van Eemeren and Rob Grootendorst-two of the earliest theorists to analyse fallacies within a dialogical context-described fallacies within only one context of dialogue, that of a critical discussion: 'A fallacy is ... defined as a speech act that prejudices or frustrates efforts to resolve a difference of opinion and the use of the term "fallacy" is thus systematically connected with the rules for critical discussion' (1995, p. 136; emphasis in original). Douglas Walton has expanded the notion of a dialogical context beyond that of a critical discussion to include dialogues like inquiry and negotiation. For Walton, shifts between different types of dialogue can generate informal fallacies: 'Some dialectical shifts... are illicit, and these illicit shifts are often associated with informal fallacies' (Walton and Krabbe, 1995, p. 102).

${ }^{39}$ Nicholas Rescher, whose aim it is to develop 'a dialectical model for the rationalization of cognitive methodology-scientific inquiry specifically included' (1977, p. xii), seeks to exclude circular sequences of argument from inquiry: "It is necessary to preclude the repetitive-indeed circular sequence: 


\begin{tabular}{|c|c|}
\hline PROPONENT & OPPONENT \\
\hline$! P$ & $+\sim \mathrm{P}$ \\
\hline$! \mathrm{P}$ & \\
\hline
\end{tabular}

[KEY: ! = categorical assertion; $+\sim=$ cautious denial]

This blockage is accomplished by adopting a special rule to proscribe the simple repetition of a previous move. The reason for such a non-repetition rule lies deep in the rationale of the process of disputation. A disputation must be progressive: it must continually advance into new terrain. Since its aim is to deepen the grounding of the contentions at issue, it must always endeavour to improve upon the reasoning already laid out, in the interests of achieving greater sophistication. Mere repetition would frustrate the aim of the enterprise' (1977, pp. 10-11; emphases in original).

40 The argument from ignorance is not just a form of presumptive reasoning in the context of dialogue. It is also an example of presumptive inference: "It is a plausible or presumptive type of inference... which rests on a major premise that is not strictly universal, but states how things normally or usually can be expected to go (subject to exceptions)... This... argumentation scheme for the argumentum ad ignorantiam has the following form.

It has not been established that all the true propositions in D are contained in $K$.

$A$ is a special type of proposition such that if $A$ were true, $A$ would normally or usually be expected to be in $\mathrm{K}$.

$A$ is in $D$.

$A$ is not in $K$.

For all $\mathrm{A}$ in D, $\mathrm{A}$ is either true or false.

Therefore, it is plausible to presume that $A$ is false (subject to further investigations in D) (Walton, 1992, p. 386; emphases in original).

${ }^{41}$ For discussion of non-fallacious circularity in economics, see Walton (1995, p. 233). Walton (1985) examines non-fallacious mathematical circularity (p. 263) and non-fallacious circular reasoning in geology and palaeontology, the latter also being examined by Rastall (1956, p. 168). In the only monograph on the argument from ignorance, Walton (1996) examines non-fallacious uses of this argument within both scientific and non-scientific inquiries.

${ }^{42}$ In doing so, we will be going against the type of view described here by Walton (1996): 'In a scientific investigation where the researchers want to "establish the facts" conclusively as a basis for building up a solid body of evidence, it may be thought desirable to avoid presumptions if at all possible. For presumptions may have to be withdrawn as further evidence builds up in the inquiry, thus necessitating revisions which could complicate a well-established theory that has been carefully constructed and drawn out' (pp. 290-291).

${ }^{43} \mathrm{Dr}$. Calman was responding to press speculation about the case of Vicky Rimmer, a 15-yearold who fell ill early in the summer of 1993 and who died on 21 November 1997. The CJD Surveillance Unit now attributes her death to CJD, but her illness did not have the characteristics of the cases now classified as nVCJD. Dr. Calman's reference to beef and hamburgers relates to claims made by Vicky's grandmother, claims to the effect that Vicky had been infected as a result of eating beef infected with BSE.

${ }^{44} \mathrm{SEAC}$ announced the link between BSE and, at the time, 10 cases of CJD in young people in the following statement of 20 March 1996: 'On current data and in the absence of any credible alternative the most likely explanation at present is that these cases are linked to exposure to BSE before the introduction of the SBO ban in 1989' (BSE Inquiry, Volume 1, p. 159).

${ }^{* 5}$ It is relevant at this stage to introduce a terminological point. Where I have been using the term "incorrect', the authors of the Inquiry Report use the term 'fallacious": "The following provi- 
sional conclusions... which were generally accepted at the time as a basis for action, were reasonable but fallacious' (BSE Inquiry, Volume 1, p. xix). I restrict the term 'fallacious' to the evaluation of reasoning at a particular point in time.

46 :Having been responsible for identifying the new disease via the network of Veterinary Investigation Centres, the CVL initiated the earliest research into BSE. Initial investigations were aimed at characterising the new disease and studying its epidemiology. So far as the former was concerned, early indications suggested that the disease was a transmissible spongiform encephalopathy (TSE), and by the beginning of June 1987 investigations had already been set in train to confirm this. These aimed to establish whether the disease was transmissible to experimental animals (hamsters were used initially), and whether SAFs [scrapie-associated fibrils], known to be associated with TSE diseases, could be detected in treated samples from brains of affected animals' (BSE Inquiry, Volume 2, p. 178).

47 'Study of the epidemiology of the disease was investigated on 3 June 1987, when Mr. Wilesmith, head of the CVL Epidemiology Department, was asked to investigate the new disease' ( $B S E$ Inquiry, Volume 2, p. 178); 'Mr. Wilesmith designed a questionnaire for use on farm visits with the object of eliciting, in the case of each casualty, any information that might have a bearing on the cause of the disease. This included exposure of individual diseased animals to various possible sources of infection such as feed, vaccines and other disease prevention methods, herbicides and pesticides, and contact with sheep. The pedigree of each animal was explored in order to see whether the disease might have a genetic source' (BSE Inquiry, Volume 3, pp. 59-60).

48 That the answer to this question is essentially indirect is demonstrated by a statement of $\mathrm{Mr}$. Bradley (Head of Pathology Department, CVL, 1983-1995; CVL's BSE research coordinator, 1987-1995) in a covering minute to Dr. Watson (Director of the CVL, 1986-1990; member of the Tyrell Committee and of SEAC). The covering minute accompanied a paper on the 'logica! approach' to BSE research and identified a number of questions to which answers were needed, one of which was 'Is BSE transmissible to primates? (\& by inference to man)'. Prior to the emergence of nvCJD in the human population an answer to the question of whether or not BSE could be transmitted to man was dependent on an inference from the results of transmission studies in primates.

${ }^{49}$ The CVL's early epidemiological studies suggested that BSE had been caused by the transmission of scrapie to cattle. However, subsequent strain-typing studies have shown this not to be the case, with the two diseases displaying different incubation periods and disease pathologies.

5: Woods and Walton (1978) couch the argument from ignorance in the language of confirmation theory as follows: 'the fallacy... consists in suppression of the possibility that $\mathrm{H}$ the hypothesis] may be unconfirmed, i.e., the live possibility that there are no known data for $\mathrm{H}$ is omitted. And in so saying, we have the suggestion ignorantiam has an epistemic aspect' (p. 91; emphases in original).

${ }^{51}$ It was only with the establishment of the Tyrrell Committee on research into spongiform encephalopathies in February 1989 that the analogy with scrapie, that had dominated much thinking about BSE, itself became the focus of research questions: 'We need to be sure that the disease really came from sheep and to know whether it is likely to establish itself long-term in bovines... If the preliminary studies and arguments-by-analogy used to determine our present control policies turn out to be incorrect, it will be essential to have well-documented facts available so that current policies can be effectively revised' (BSE Inquiry, Volume 1, p. 56).

52 'The possibility that maternal transmission played a role in transmission was considered early in the epidemic. There was evidence of maternal transmission of scrapie in sheep but not in other TSEs such as kuru, non-familial CID and transmissible mink encephalopathy (TME). It was therefore essential to determine if it occurred in cattle, as procedures put in hand for arresting the epidemic depended on maternal transmission not being an important factor. Lateral transmission was also considered since this, too, had been identified as a transmission route for scrapie' (BSE 


\section{Louise Cummings}

Inquiry, volume 2, p. 95).

${ }^{32}$ In a written statement to the Inquiry Ms. Carol Richardson (Senior Pathologist at CVL) said of cow 142 that she had examined: 'Although I had never seen this type of lesion before in a cow I had frequently seen the combination of neuronal and neuropil vacuolation with this distribution in Scrapie. To me, this was Scrapie in a cow' (BSE Inquiry, Volume 3, p. 6). Cow 142 was the first live animal to be sent to the CVL for euthanasia and post-mortem examination. Subsequent histopathological studies have established different patterns of vacuolation in scrapie and BSE.

${ }^{54} \mathrm{I}$ argued earlier in the main text that from the outset of inquiry into BSE, various biological studies indicated essential dissimilarities between BSE and scrapie. These dissimilarities, I contended, invalidated the very basis of an analogy with scrapie, resulting in the fallacy of false analogy. The process of invalidation that is involved in this case is similar to that which is being considered in the main text.

${ }^{\$ 3}$ Inquiry witness John Collinge is Professor of Molecular Neurogenetics, Imperial College School of Medicine at St. Mary's Hospital in London. He has been a member of SEAC since December 1995.

${ }^{56}$ Such was the case, for example, when the first oral transmission of BSE to mice was achieved and when the first primate-a marmoset - succumbed to BSE through cerebral inoculation. In relation to the former transmission, a MAFF press release of 1 February 1990 stated: 'The BSE results therefore provide further evidence that BSE behaves like scrapie, a disease which has been in the sheep population for over two centuries without any evidence whatsoever of being a risk to human health' (BSE Inquiry, Volume 1, pp. 127-128). Of the latter transmission the Inquiry Report records: 'On I4 February 1992 BSE was found to have been successfully transmitted to a marmoset by cerebral inoculation. This was the first transmission to a primate. A meeting of SEAC was immediately called to consider the implications of this. SEAC concluded that as marmosets had in the past been infected with SEs, including scrapie, using similar methods, the results were not surprising and had no implications for the safeguards already in place for human and animal health' (Volume 1, p. 139).

${ }^{57}$ Rescher (1979) describes this Euclidean model as follows: 'Certain theses are to be basic or foundational: like the axioms of geometry, they are to be used to justify other theses without themselves needing or receiving any intrasystematic justification. A part from these fundamental postulates, however, every other thesis of the system is to receive justification of a rather definite sort. For every nonbasic thesis is to receive its explanation along an essentially linear route of demonstration (or derivation or inference) from the basic theses that are justificationexempt or self-justifying. There is a step-by-step, recursive process-first of establishing certain theses by immediate derivation from the basic ones, and then of establishing further theses by sequential derivation from already established theses. Systematization proceeds in the manner characteristic of axiomatic systems' (pp. 40-41).

"Rescher $(1979$, p. 96) represents the retrospective reappraisal of datahood in his coherentist epistemology as follows:

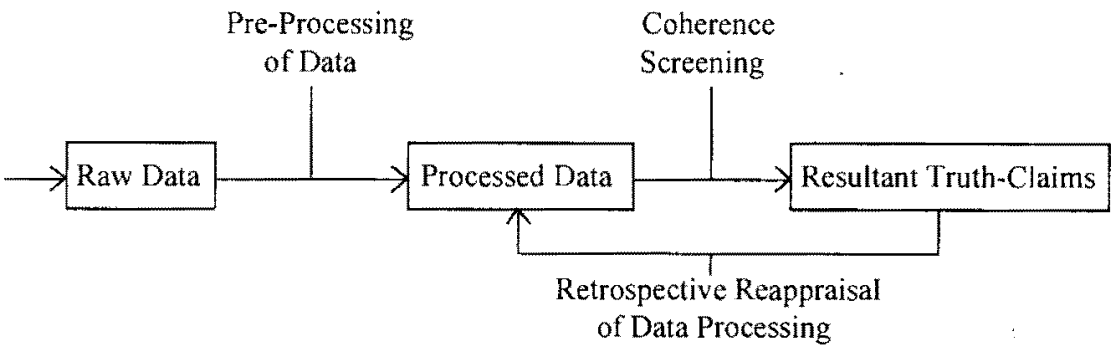


Rescher contends that there exists 'a cyclic movement, a closing of the cycle that requires a suitable meshing - a meshing process that should eventually retrovalidate (retrospectively revalidate) the initial criteria of datahood with reference to the results to which they lead' $(1979, \mathrm{p}$. 95).

${ }^{39}$ In the main text 1 am using the term 'standard' of pre- and post-1970 theorising about the informal fallacies-even though this term has come to be identified through the work of Charles Hamblin with pre-1970 theorising-as the single-argument analysis that I am taking to be definitive of standard theorising is typical of the work of many post-1970 theorists (e.g., John Woods and Douglas Walton).

${ }^{67}$ It is interesting to note that for van Eemeren and Grootendorst, a pragma-dialectical analysis of the fallacies reveals essential similarities between certain fallacies and provides a more systematic, less ad hoc analysis of the fallacies, both of which I am claiming to be features of my own analysis: 'This brief overview may suffice to show that the pragma-dialectical analysis of the traditional fallacies as violations of the rules of critical discussion is more systematic than the Standard Treatment. Instead of being given ad hoc explanations, all the fallacies fall under one or more of the rules for critical discussion. A comparison between the violations of the pragmadialectical rules and the traditional categories also shows that the pragma-dialectical analysis is more refined. Fallacies that were only nominally lumped together are now either shown to have something in common or they are clearly distinguished, and genuinely related fallacies that were separated are brought together' (1995, p. 142).

61 'Taken together, these rules [rules for the performance of speech acts] constitute a theoretical definition of a critical discussion' (van Eemeren and Grootendorst, 1995, p. 135).

\section{References}

Brown, P., Cathala, F., Raubertas, R.F., Gajdusek, D.C. \& Castaigne, P. (1987). The Epidemiology of Creutzfeldt-Jakob disease: Conclusion of a 15-Year Investigation in France and review of the world literature. Neurology 37, pp. 895-904.

$B S E$ Inquiry (2000). Volume 1: Findings and conclusions. The Stationery Office.

BSE Inquiry (2000). Volume 2: Science. The Stationery Office.

BSE Inquiry (2000). Volume 3: The Early Years, 1986-96. The Stationary Office.

BSE Inquiry (2000). Volume 4: The Southwood working party, 1988-89. The Stationery Office.

BSE Inquiry (2000). Volume 5: Animal health, 1989-96. The Stationery Office.

Collins, A., Aiello, N., Warnock, E.H.\& Miller, M.L. (1975). Reasoning from Incomplete Knowledge. In D.G. Bobrow \& A. Collins (Eds), Representation and understanding: studies in cognitive science. New York: Academic Press.

Copi, I.M. (1961). Introduction to logic. $2^{\text {nd }}$ edition. New York: Macmillan.

Copi, 1.M. (1972). Introduction to logic. 4'h edition. New York: Macmillan.

Hamblin, C.L. (1970). Fallacies. London: Methuen.

Rastall, R.H. (1956). Geology. Encyclopaedia Britannica 10, pp. 168-173.

Rescher, N. (1977). Dialectics: A controversy-oriented approach to the theory of knowledge. Albany, NY: State University of New York Press. 


\section{Louise Cummings}

Rescher, N. (1979). Cognitive systematization: A systems-theoretic approach to a coherentist theory of knowledge. Oxford: Basil Blackwell.

Rescher, N. (1987). Forbidden knowledge and other essays on the philosophy of cognition. Dordrecht: Reidel.

Robinson, R. (1971). Arguing from ignorance. The Philosophical Quarterly 21( 83), pp. 97-108.

Schedler, G. (1980). The argument from ignorance. International Logic Review 11, pp. 66-71.

Van Eemeren, F.H. \& Grootendorst, R. (1995). The Pragma-Dialectical approach to fallacies. In H.V. Hansen \& R.C. Pinto (eds.), Fallacies: Classical and contemporary Readings. University Park, PA: The Pennsylvania State University Press.

Walton, D.N. (1985). Are circular arguments necessarily vicious? American Philosophical Quarterly 22(4), October, pp. 263-274.

Walton, D.N. (1989). Informal Logic: A handbook for critical argumentation. Cambridge, UK: Cambridge University Press.

Walton, D.N. (1990). What is reasoning? What is an argument? The Journal of Philosophy 87, pp. 399-419.

Walton,D. (1992). Nonfallacious arguments from ignorance. American Philosophical Quarterly 29(4), pp. 381-387.

Walton, D.N. (1995). The Essential ingredients of the fallacy of begging the question. In H.V. Hansen \& R.C. Pinto (eds.), Fallacies: Classical and contemporary readings. University Park, PA: The Pennsylvania State University Press.

Walton, D.N. (1996). Arguments from ignorance. University Park, PA: The Pennsylvania State University Press.

Walton, D.N. \& Krabbe, E.C. (1995). Commitment in dialogue: Basic concepts of interpersonal reasoning. New York: State University of New York Press.

Will, R.G. (1993). Epidemiology of Creutzfeldt-Jakob disease. British Medical Bulletin 49(4), pp. 960-970.

Woods, J. \& Waiton, D.N. (1978). The fallacy of 'ad ignorantiam'. Dialectica 32(2), pp. 87-99.

Louise Cummings

Department of English and Media Studies Nottingham Trent University Clifton Campus, Clifton Lane Nottingham, NGH 8 NS England, United Kingdom louise.cummings@ntu.ac.uk 